\title{
Experiments and Fault Analysis of Low Voltage Hydroelectric Station in Microgrid
}

\author{
Yi Yang ${ }^{1, ~ a ~}$, Engang Tian ${ }^{1, ~ a ~}$, Jingsong Chen ${ }^{2, \mathrm{~b}}$, Xiaohui $\mathrm{Li}^{1, \mathrm{a}}$ and Huaren $\mathrm{Wu}^{1, \mathrm{a}}$ \\ ${ }^{1}$ School of Electrical and Automation Engineering, Nanjing Normal University, Nanjing 210042, \\ China; \\ ${ }^{2}$ Talent science and technology Co., LTD, Nanjing 211800, China. \\ ayy21090319@163.com, bjesoon@gmail.com
}

Keywords: Hydroelectric station, experiment, fault analysis, microgrid

\begin{abstract}
Different from the wind energy and solar energy, the hydro-generator is not intermittent energy, which can overcome the disadvantages of wind-mill generator and PV array in microgrid. This paper describes the static experiments, dynamic experiments and the grid-connected tests of low voltage hydroelectric station and analyzes the faults of the generator excitation system. Some reasons about the failure of the generator starting are described in detail and the corresponding treatment methods are given. The problems of synchronous close of circuit breaker are analyzed and the solutions are also provided. The experiments and the fault analysis depicted in the paper make contributions to solve electrical technology problems of low voltage hydroelectric station for technician, which does good to the safety operation of the stations and microgrid.
\end{abstract}

\section{Introduction}

As the rapidly growing of the ecological civilization construction, our country puts more and more emphasis on the development of the hydroelectric stations. Now, the construction and retrofit of the low voltage hydroelectric station have been put in a key position. Up to now, there are more than 30,000 rural low voltage hydroelectric stations in the country. Most of them have been used for over 30 years since 1960s and can hardly make any equipment update, maintenance and technical innovation [1, 2]. For the seriously aged facilities and equipment in the stations, the potential security problems are increasingly prominent [3]. In addition, the equipment failures can cause other problems such as low automation, low running efficiency, more human and material resources consuming and even threaten the safety of people's lives and property [4].

Based on the reasons mentioned above, Chinese government has made great efforts to undertake the synergism expansion transformation. In the synergism expansion, we adopt the integration technology of automation and control, which is an effective measure for promoting energy saving, reducing security risks.

Consequently, for purpose of more efficient synergism expansion, the electrical commission and related methods for fault treatment will become even more significant.

\section{Experiments of low voltage hydroelectric station}

Before putting the low voltage hydroelectric station into operation, we must perfect the work of electrical commissions that contains check-up before energization, static simulation, dynamic simulation, the grid-connected test and the trial operation.

\subsection{Check-up before energization}

Before energization, we must check up whether the power circuit is short circuited or open circuited first. If there is no mistake, then we can do the next experimentation.

\subsection{Static experiments}

Static simulation experimentation means we test the integrative control cabinet independently under the condition that the generator and other equipment are not running, which is aiming to verify grid-connected function of the integrative control cabinet. 
There are several steps to complete the experimentation. First, we should switch off the outdoor breaker. Referring to Fig. 1, we should separate disconnector (QS) and breaker (QF) in the control cabinet to ensure that the system side of the control cabinet disconnects with the outside electric power line, then take apart the wiring from A1, B1, C1 and N1 which are connected with generator. Second, we may use wire to connect A with A1, B with B1, C with C1, and N with N1 and connect all of them with power distribution cabinet beside the control cabinet and use 380V three-phase power. If the station site doesn't have power distribution cabinet, we can use the three-phase power from outside line, which has the same functionality.



Fig. 1 Interior view of integrative control cabinet [5]

If all the preparatory work has been completed, we should setup the control cabinet's parameter, and press start button.

After starting, we should observe whether the data in the display instrument is normal, if the data has been confirmed correct, then press switching button and opening button. The correctness of the switch-on-off shows that synchronism check and integrative control cabinet is in good condition and is suitable to do the next experimentation.

\subsection{Dynamic experiments}

Dynamic simulation experimentation, which is commonly known as pseudo synchronous test, means a test of synchronous grid connected on the premise of separating disconnector (QS) in the integrative control cabinet.

The steps of the dynamic simulation experimentation are as follows. First, dismantle and recover wires which are connected when we do static simulation experimentation. Second, separate disconnector (QS) in the cabinet, close outdoor disconnector (QS) and outdoor circuit breaker (QF). The system side of the control cabinet takes power from outside electric power line. Connect cabinet's A1, B1, C1 and N1 with generator's U, V, W and N. Ensure that the positive and negative poles of the excitation circuit are connected properly.

After everything is ready, we carry out the dynamic simulation experimentation by manual mode first. The control cabinet will be set up in a way to track the power grid voltage. Then we press the start button and the control cabinet begins to collect the valve signal. When the valve is detected fully to be opened, the machine will be turned on and the governor will work. Then the hydroturbine rotates to drive the generator to run. In this process, there must be someone beside the governor to observe its running status. Whenever something is wrong with the governor, it should be shut down immediately to prevent the occurrence of accidents (such as over speed). Once the detected generator-frequency reaches $48 \mathrm{~Hz}$, button for manual initiation of excitation should be pressed, and then the machine starts excitation and builds up voltage. The voltage will eventually be stable at about $400 \mathrm{~V}$. At this point, the circuit breaker begins to store energy. When breaker storage is completed, the cabinet will prompt closing. At this time, pull down the switch handle, and the control cabinet will automatically collect voltage from both system side and the generator side. Meanwhile, the cabinet will check synchronization. When the voltage difference and the frequency difference meet the requirement, the integrative cabinet will send control commands to close circuit breakers. At this time, the data in the display instrument should be observed.

Before the synchronization instruction is issued in the manual mode process, we need to detect 
the three-phase voltage sequence artificially form generator and system side. There are three main methods to measure the phase sequence, but normally we use the phase-sequence meter, which means when the buildup of voltage has been completed, we use phase-sequence meter to detect 3 phase voltage sequence respectively. If the related lights in phase-sequence meter are positive rotation, which means voltage is in the same sequence.

When all above experiments are detected accurately, we can press the stop button to shut down the generator. It is forbidden to pull down the switch handle directly to shut down the generator. When the generator is shut down and become complete static, switch automatic-manual- handle to automatic mode. After that, press start button and observe the digital display meter, just like what we have done in above operation. If every experiment works well without any problem, it shows that we have successfully completed the dynamic simulation experimentation.

\subsection{Grid-connected test}

After the test of static simulation, dynamic simulation and confirming the whole systems to be correct, we can carry out the grid connection test.

The method of grid connection test is to close disconnector (QS) in the cabinet, and close power line disconnector (QS) as well as power line breaker (QF) to ensure the voltage in system side of cabinet is from power grid. Then according to the flow of the dynamic simulation test, do the manual mode test first, automatic mode test second. When the circuit breaker of the cabinet can close correctly in the course of above, it means we realize grid-connected generation successfully. After the success of the grid-connected, we can rotate capacity control knob to increase or decrease the active power and reactive power so that we can know whether the system's load capacity is equal to meeting our requirements.

In this position, we should test the performance about relevant protection, such as load rejection protection, over-voltage protection, over-current protection, over-speed protection, loss of excitation protection and excitation overload protection.

Load rejection is the most common in hydroelectric station. So it is very important.

Load rejection protection means when it encounters the grid fault, the main switch of generator trips, and a large amount of excess energy will make hydro-generator's speed rise rapidly. In order to avoid the adverse consequences caused by the sharp rise in speed, it is necessary to be protected.

In load rejection protection, if there is no special requirement, we usually carry out four tests divided by active power into $25 \%, 50 \%, 75 \%$ and $100 \%$. For example, we conduct a load rejection protection of 50\%-active-power. When we realize grid-connected generation successfully, adjust the active power to half of full power, and then press emergency shutdown button. At this time, system will remove all the active power which can lead to the generator's frequency rise rapidly. If the frequency reaches the setting value of the protection, the breaker trips to shut down the generator. If everything is ok, this experiment is completed.

\section{Fault analysis}

In the course of electrical commissions, there is little chance that everything goes smoothly. We will meet some troubles inescapability. At present, the integrative control cabinet is widely used in the hydro-generator unit with a capacity of $1000 \mathrm{~kW}$ and below. And the integrative control cabinet mainly contains the following eight functions, namely the automatic control of the unit startup and shutdown, generator excitation control, the speed control of hydraulic turbine, quasi-synchronizing closing, generator relay protection, digital display instrument, automatic-economic power generation and unit state monitoring. The controller provides RS485 communication interface to realize data sharing so that we can make generator unit's remote control and centralized control come true. In the following, I will briefly explain the problems we may meet in the electrical commissions [6].

\subsection{Failing in starting excitation}

If we fail to start excitation in electrical commissions, the possible reasons are:

1) New units need to store magnetic energy in order to start excitation. When generator comes to rated speed, and its voltage ranges from $8 \mathrm{~V}$ to $10 \mathrm{~V}$, then we do not need to store magnetic energy. 
2) FU8 may break down in the excitation circuit of the control cabinet. (Referring to Fig. 2)

3) Check the control cabinet to know whether the diode is inversely connected. (Referring to Fig. 2)

4) If the generator is a no-brush generator set, there may miss connecting $\mathrm{N}$ from generator to excitation component perhaps. At this moment, we must distinguish the different between brushless excitation component and brush excitation component. In PAS661 A, $75 \mathrm{mV}$ of brushless excitation components is corresponding to $20 \mathrm{~A}$, while it is $300 \mathrm{~A}$ for brush excitation component.

5) Check whether the excitation circuit is closed, and whether the wiring is correct. New units need not to distinguish the positive and negative of excitation while old units need.

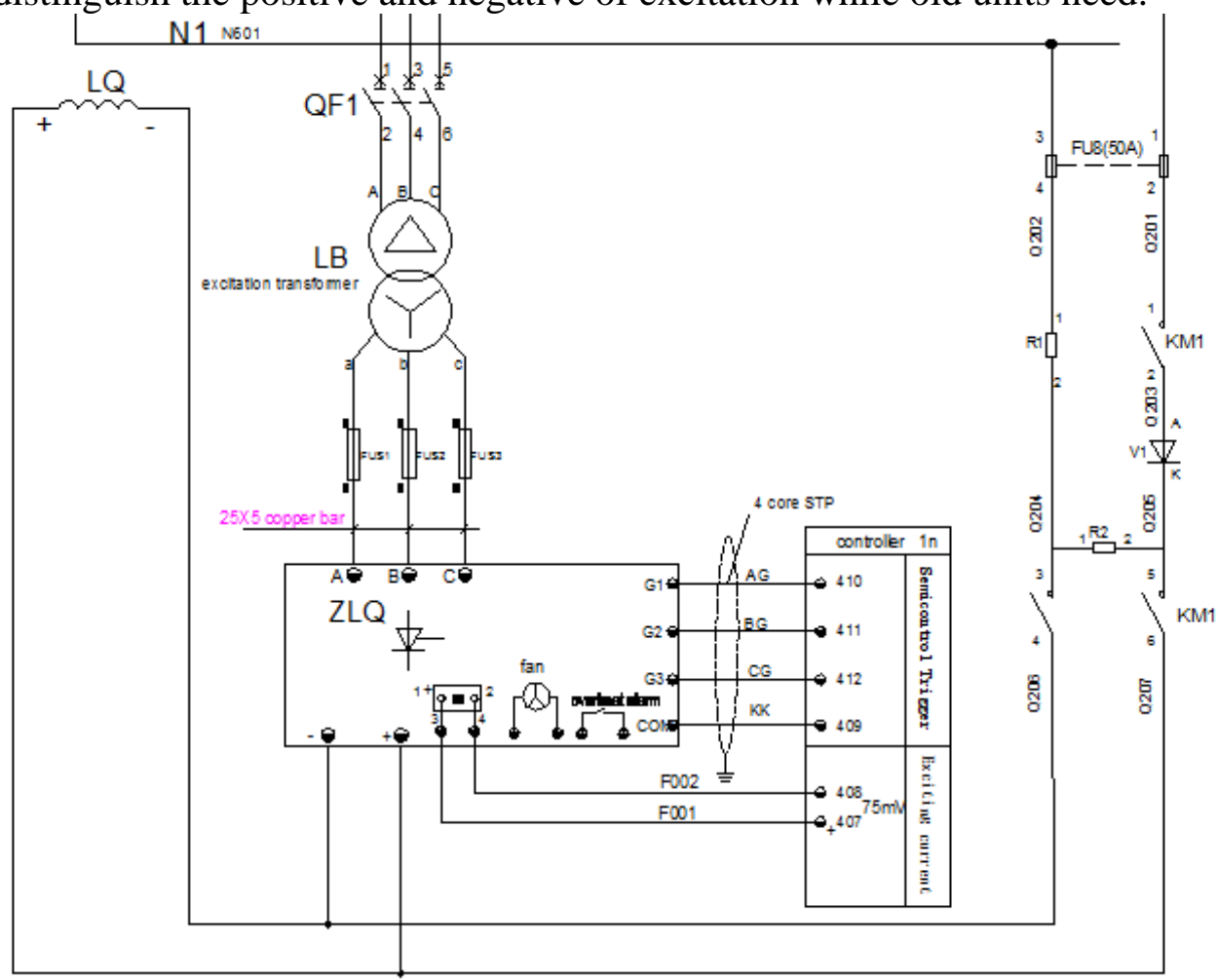

Fig. 2 Excitation circuit diagram [5]

\subsection{Closing fault in the low voltage hydroelectric station}

In the experiments of low voltage hydroelectric station, some closing fault would be met.

1) After establishing voltage, the breaker comes to close in asynchronous.

Reasons: When the units come to establish voltage, there may have high voltage in the generator which can break down the varistor in closing circuit. The closing circuit will be in short circuit and make the breaker comes to close in asynchronous.

Methods: Remove the varistor.

2) The breaker fails to close while there has voltage and signal in closing outlet. Actually, the breaker does nothing.

Reasons: According to the phenomenon we stated above, we can judge that the breaker closing coil is burnout.

Methods: Replace the closing coil of the circuit breaker.

3) When grid-connected is completed, excitation current fluctuates range from 0 to $4 \mathrm{~A}$, and maintains at 0 for long time. Besides, reactive power increases automatically.

Reasons: Excitation current line of current diverter is inversely connected which can fluctuate reactive power.

Methods: Exchange the excitation current lines which are connected to 407 and 408. (Referring to Fig. 2)

\section{Conclusion}

Microgrid is going to play an extremely important role in distribution system. As the important 
component of microgrid's distributed generation, normal operation of low voltage hydroelectric station cannot be ignored. The process of electrical commissions said in this article is the basic debugging process in low voltage hydroelectric station. It can help us complete the debugging work scientifically and reliably.

Certainly, in the process of electrical commissions will encounter some problems inevitably. What we analyzed in this article about the reasons and methods of failing in starting excitation can help us to solve the fault, so the generator can start excitation normally. While when the generator succeeds in starting excitation, there may be many problems in the synchronous closing to prevent grid-connected. What we introduced in this article about the reasons and methods of failing in the synchronous closing can help us to solve the fault, so we can realize synchronous closing. After that, the low voltage hydroelectric station's electrical commissions can be completed in time. Then it can be put into operation timely which can take great contributions to the development of microgrid.

\section{Acknowledgements}

This work was financially supported by the National Natural Science Foundation of China (51177074).

\section{References}

[1] Renwen Lu, Automatic control system for less person on duty of 400V low voltage unit, Small Hydro power, 4 (2013), 79-82.

[2] Xing Wu, Xiaogang Yin, Xi Song, Jing Wang, Research and application status of microgrid technology in China, High Voltage Apparatus, Vol.49 No.9 (2013) 142-149.

[3] Li Yan-pin, Chang Ji-yuan, Small Hydropower Station Monitoring System Structure Analysis, Electrical and Control Engineering (ICECE), 2010 International Conference on, IEEE Conference Publications, pp.1965-1967 (2010).

[4] Hasan H.Coban, Renata Varfolomejeva, Antans Sauhats, Inga Umbrasko, Small Hydropower Plants Operations Optimization in the Market Conditions, Information, Electronic and Electrical Engineering (AIEEE), 2014 IEEE 2nd Workshop on Advances in, IEEE Conference Publications, pp.1-5 (2014).

[5] Talent science and technology Co., LTD, Electrical design document of the integrative control cabinet (2013).

[6] Talent science and technology Co., LTD, Instruction V1.0 about PAS661A generator self-motion control device (2013). 